\title{
INFLUENCE OF FFL-13 OIL ON PSA STAPLE FIBER PROPERTIES
}

\author{
Haokai Peng ${ }^{1,3}$, Guo Zheng ${ }^{1,2,}$, Yu Sun ${ }^{2}$, and Rui Wang ${ }^{1}$ \\ ${ }^{1}$ Tianjin Polytechnic University, School of Textiles, Tianjin, 300387, China, 399 Binshui West Road, XiQing District \\ ${ }^{2}$ Tianjin Polytechnic University, Textile Agents CO., Ltd., Tianjin, 300270, China, 915 Jinhui Road, DaGang District \\ ${ }^{3}$ Tianjin Polytechnic University, School of Textiles, Innovation Platform of Intelligent and Energy-Saving Textiles, Tianjin, 300387, China, 399 Binshui West \\ Road, XiQing District \\ E-mail:zhengguo0703@126.com
}

\begin{abstract}
:
With the aim of enabling the easy spinning of polysulfonamide (PSA) fibers, FFL-13 oil was successfully prepared. Specific resistance, the coefficient of friction, carding machine, electrostatic, cohesion, scanning tunneling microscopy (STM), and Fourier transform infrared (FTIR) spectroscopic analyses were conducted in this study. Results showed that increase in oil concentration, ambient temperature, and humidity induces decrease in PSA fiber-specific resistance and gradual increase in the antistatic property. Relatively high oil concentration and ambient humidity may cause fibers to become sticky and show increased roller winding. As oil concentration increased, fiber cohesion increased and fiber gliding properties showed an initial increase and subsequent decrease. The ideal oil concentration was determined to be $0.6 \%$. This study proves that STM may effectively be used to determine the optimal oil concentration.
\end{abstract}

\section{Keywords:}

Polysulfonamide staple fiber, Oil concentration, Specific resistance, Friction coefficient, STM

\section{Introduction}

Polysulfonamide (PSA) is a high-temperature-resistant fiber with independent intellectual property rights owned by the Chinese. Figure 1 illustrates the chemical structure of PSA fibers. Given the additional sulfone group $\left(-\mathrm{SO}_{2}-\right)$ structure in its main molecular chain and the existence of conjugated aromatic rings, the electron density of the amide is significantly reduced and the PSA fiber cannot be destroyed at high temperature [1,2]. PSA fibers possess numerous desirable properties, such as high tensile strength and modulus, flame resistance, chemical corrosion, resistance to high temperature, electrical insulating properties, and anti-electromagnetic radiation [3]. Therefore, this fiber can be applied to several areas, such as metallurgy, fire control, military projects, and space navigation [4]. Jia et al. recently investigated the ablation and thermal properties of ethylene-propylene-diene elastomer composites reinforced with PSA short fibers $[5,6]$. However, PSA presents a high initial modulus, mass-specific resistance, a relatively low curling stability, and friction, all of which contribute to its weak spinnability. Key problems such as strong static, weak cohesive forces among fibers, difficulties in the resultant yarn, and increase in hairiness, especially in long strands, have been observed during the spinning process of PSA [7]. These problems exert a direct effect on the quality of the resultant yarn [8-10]. Thus, developing an appropriate fiber oil that provides fibers with strong antistatic properties and ideal friction performance is urgently needed to smoothen the spinning process [11-15].

Multiple research studies on the performance of different staple fiber oils have been reported recently $[16,17]$. However, no studies have focused on the spin finish on PSA fibers. In this study, we developed a PSA staple fiber (FFL-13) oil that decreases the specific resistance of fibers and increases the cohesion and spinning of PSA fibers. Using Photoshop and MATLAB software to assess scanning tunneling microscopy (STM) images and to determine the reasonable oil concentrations is an innovative method. Image enhancement may be used to achieve stereoscopic effects and directly observe oil spread on the fiber surface. Findings obtained through this method may provide valuable guidance in choosing reasonable oil concentrations during the spinning process.<smiles>CC(C)OC(=O)c1ccc(C(=O)Nc2cccc(S(=O)(=O)c3cccc(NCC(C)(C)C(=O)c4ccc(C(=O)Nc5ccc(S(=O)(=O)c6ccc(N(C)C)cc6)cc5)cc4)c3)c2)cc1</smiles>

Figure 1. The chemical structure of PSA fiber 


\section{Materials and Methods}

\subsection{Materials}

The PSA fiber used in this experiment was provided by Shanghai Tanlon Fiber Co., Ltd. and featured a fineness of 2.3 dtex. The acetone was provided by Tianjin Chemical Reagent Factory (China). 80G33NF (main chemical compositions are C12 phosphate and emulsifiers) was provided by Schill \& Seilacher Company, Germany. FFL-13 oil (C12 phosphate, C8 phosphate, $\mathrm{C} 12$ ester-ether and emulsifiers) was provided by Tianjin Polytechnic University Textile Agents Co., Ltd., China.

\subsection{Preparation of PSA fibers}

PSA fibers were washed in acetone for $30 \mathrm{~min}$ to remove surface contaminants, thoroughly rinsed with distilled water, and then dried in a vacuum for more than $12 \mathrm{~h}$ to eliminate the remaining acetone.

Identical amounts of PSA fiber samples were obtained and immersed in FFL-13 oil at concentration levels of $0.2 \%, 0.4 \%$, $0.6 \%, 0.8 \%$, and $1 \%$ or $80 \mathrm{G} 33 \mathrm{NF}$ at a concentration of $0.6 \%$ for $30 \mathrm{~min}$. The pretreated PSA fibers were then dried in an oven for $2 \mathrm{~h}$ at $80^{\circ} \mathrm{C}$.

\section{$\underline{2.3 \text { Specific resistance measurements }}$}

The specific resistance of the fibers was measured using a fiber resistance meter (YG321, Changzhou Textile Instruments Factory Co., Ltd., China) according to GB/T 14342-2015. PSA fibers were balanced for $2 \mathrm{~h}$ at $25^{\circ} \mathrm{C}$ and $60 \%$ relative humidity $(\mathrm{RH})$.. Twenty measurements were performed for each sample. The formula used is

$$
\rho^{v}=R \cdot \frac{m}{L^{2} \cdot d}
$$

where $\rho^{v}$ is the specific resistance $(\Omega \cdot \mathrm{cm}), R$ is the measuring resistance $(\Omega), m$ is the fiber quality $(\mathrm{g}), L$ is the distance between the two plates $(\mathrm{cm})$, and $d$ is the fiber density $\left(\mathrm{g} / \mathrm{cm}^{3}\right)$.

\subsection{Friction coefficient measurements}

A yarn friction coefficient tester (Y151, Changzhou Textile Instruments Factory Co., Ltd, China) was used to measure the static and dynamic friction coefficient between fiber and metal (F/M) and between fibers (F/F). The dynamic friction test speed was $30 \mathrm{r} / \mathrm{min}$, and the wrap angle was $180^{\circ}$ at $25^{\circ} \mathrm{C}$ and $60 \% \mathrm{RH}$ [18]. Thirty measurements were performed for each sample.

\section{$\underline{2.5 \text { Carding performance measurements }}$}

A carding testing machine (AS181A, Jiangxi, China) was used to comb the PSA fibers at $25^{\circ} \mathrm{C}$ and $60 \% \mathrm{RH}$. An electrostatic analyzer (JFY-VR-2, China) was used to measure the dynamic voltage during the carding process [12].

\subsection{Cohesion measurements}

We used a cohesion machine (INSTON-4501, America) to measure the cohesive force of the fiber web after carding at $25^{\circ} \mathrm{C}$ and $60 \% \mathrm{RH}$. The fiber webs were cut into $250 \mathrm{~mm} \times$ $125 \mathrm{~mm}$ pieces and were placed on the machine. The ratio of maximum load to weight was the cohesion [12, 19]. Twenty measurements were performed for each sample.

\subsection{Surface morphological observation}

An STM (STM.IPC-205B, Chongqing University) was used to observe the surface morphology of the fibers. Photoshop and MATLAB software were used to analyze and process the grayscale figures.

\subsection{Fourier transform infrared spectroscopic analysis}

The chemical structures of FFL-13 and 80G33NF were characterized using Fourier transform infrared (FTIR) spectra (FTIR-650, China) obtained from $\mathrm{KBr}$ pellets over the wavelength range of $400 \mathrm{~cm}-1$ to $4000 \mathrm{~cm}-1$.

\section{$\underline{\text { 2.9 Statistical analysis }}$}

Tukey's test and one-way analysis of variance were used to compare the specific resistances, friction coefficients, dynamic voltages, and cohesion of the fibers [20]. P-values less than 0.05 were considered significant.

\section{Results and discussions}

\subsection{Effect of the FFL-13 oil concentration on the fiber antistatic property}

Fiber oil concentration exerts a significant influence on specific resistance. Figure 2 shows that the specific resistance of PSA fibers without oil reached $10^{14} \Omega \cdot \mathrm{cm}$ at $25^{\circ} \mathrm{C}$ and $60 \%$ $\mathrm{RH}$. Specific resistance decreased as the oil concentration increased. This result may be explained by the fact that hydrophilic groups in the fibers increase as the oil quantity increases, thereby resulting in enhanced electroconductibility and decreased specific resistance in the fibers. The antistatic principles of chemical fiber oil show a close relationship with its adsorption method on the fiber surface. The fiber surface adsorbs hydrophobic groups. When hydrophilic groups are exposed to air, they form a hydrophilic film, which causes difficulties in producing static. A watercourse is then formulated after the hydrophilic film absorbs air moisture, during which the static generated is easily transmitted to the atmosphere, which produces antistatic effects [21]. The fibers exhibit poor antistatic properties when the oil concentration is as low as $0.2 \%$ and the specific resistance of fiber is around $10^{9} \Omega \cdot \mathrm{cm}$. When the oil concentration is $0.4-0.6 \%$, the specific resistance of fiber is approximately $10^{7} \Omega \cdot \mathrm{cm}$. Although the antistatic properties of fiber are further enhanced when the oil concentration is as high as $0.8 \%$ or $1 \%$, high oil concentrations could lead to problems such as increases in oil film thickness on the surface of the fibers, sticky fibers, intertwined roller, and difficulties in textile processing. Therefore, applying PSA short fiber oil at a concentration of around $0.6 \%$ is ideal. 


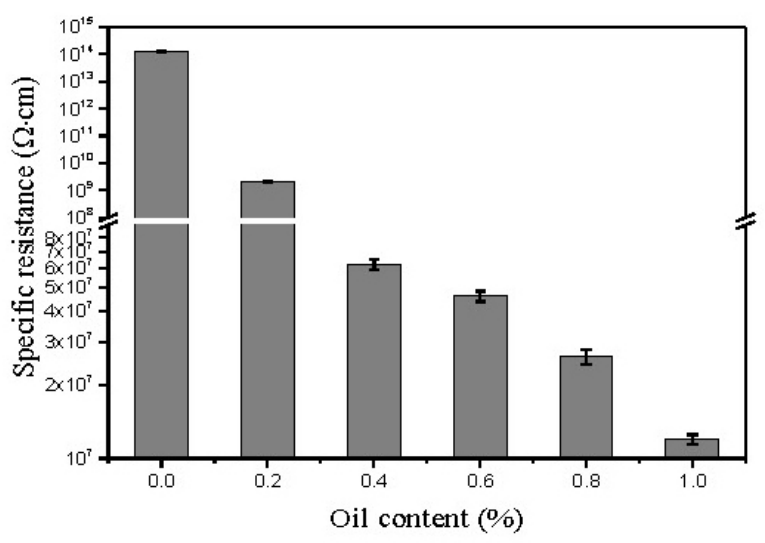

Figure 2. Effect of oil concentration on specific resistance of PSA fiber

\subsection{Effect of environmental conditions on the antistatic property of fiber}

Figures 3 and 4 show that when the FFL-13 oil concentration is $0.6 \%$, the impact of humidity on the antistatic property of fiber is more extensive than that of temperature. The specific resistance of fiber decreased as the environmental temperature and humidity increased. Specific resistance of fiber showed declines and antistatic properties of fiber increased when the fiber oil concentration and environmental temperature were at similar levels and when humidity increased. This result may be explained by the fact that relatively high environmental humidity increases air ionization and fiber static charges are neutralized with the air opposite ion, which accelerates the static attenuation. Although excessive hygroscopic fibers can improve fiber antistatic properties by increasing humidity, these fibers are inclined to stick together, which affects their spinnability. Therefore, humidity control must consider the various elements [22].

\subsection{Effect of the FFL-13 oil concentration on the fiber friction coefficient}

This experiment discusses the influence of fiber oil on the fiber friction performance by testing the friction coefficient of fibers containing different oil concentrations. The corresponding interrelationships between fiber and metal (F/M) and between fibers $(F / F)$, static friction coefficient $(\mu s)$, and dynamic friction coefficient $(\mu \mathrm{d})$ are shown.

Figure 5 shows that with increasing oil concentration, F/ M $\mu$ s and F/M $\mathrm{fd}$ first increase and then decrease. This phenomenon means the gliding property of the fibers first increases and then decreases. The gliding property shows ideal levels when the oil concentration is $0.6 \%$ because the fiber oil is unable to evenly coat the fiber surface at other oil concentrations, such as $0.4 \%$. This phenomenon leads to fiber surface roughness, which induces large values of $F / M \mu s$ and $\mathrm{F} / \mathrm{M} \mu \mathrm{d}$. By contrast, the fiber oil evenly spreads out the fiber surface at a concentration of $0.6 \%$, thereby causing declines in $\mathrm{F} / \mathrm{M} \mu \mathrm{s}$ and $\mathrm{F} / \mathrm{M} \mu \mathrm{d}$ while improving fiber gliding properties. As

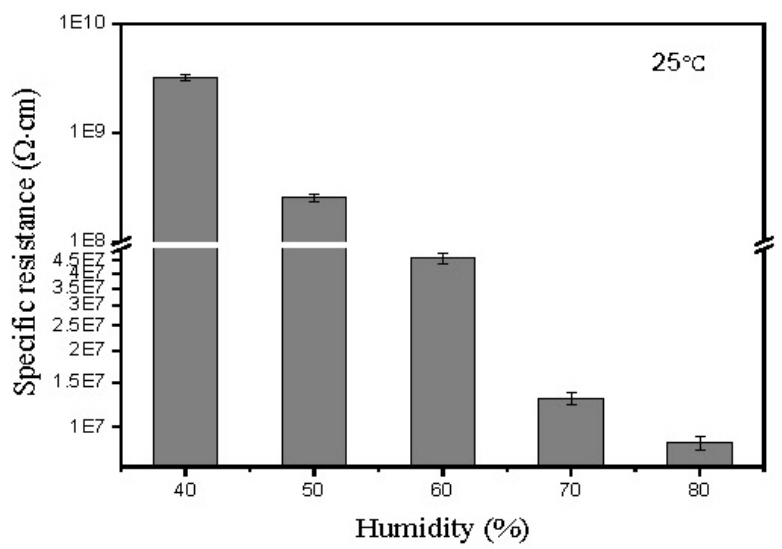

Figure 3. Effect of humidity on specific resistance of PSA fiber

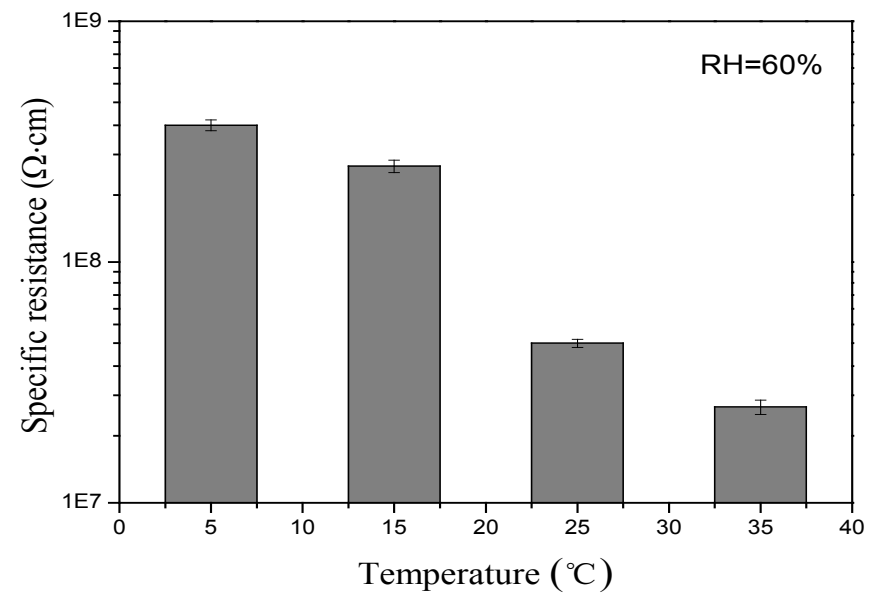

Figure 4. Effect of temperature on specific resistance of PSA fiber

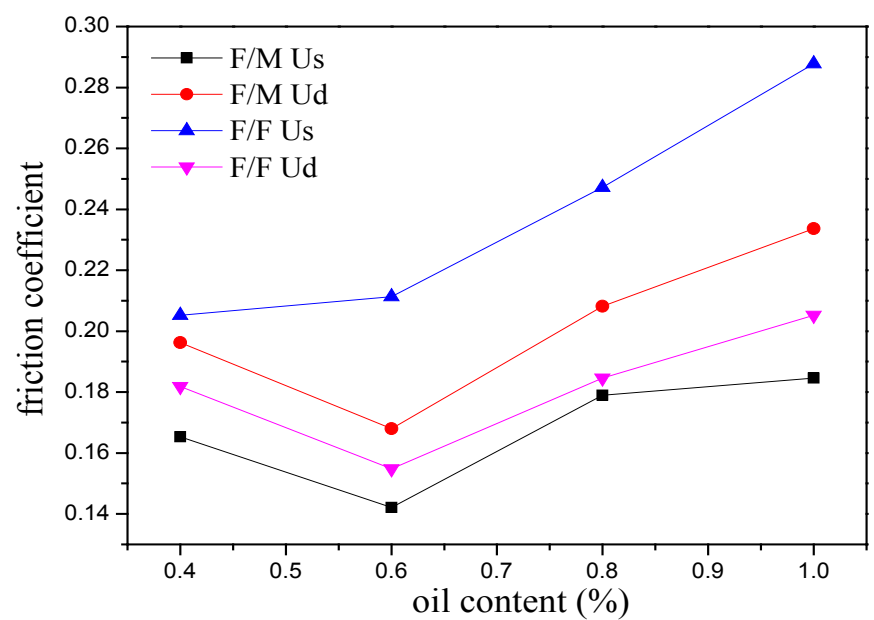

Figure 5. Effect of the oil concentration on friction coefficient of PSA fiber

oil concentration increased, the $D$-value between $F / F \mu s$ and $\mathrm{F} / \mathrm{F} \mu \mathrm{d}$ increased when the fiber cohesion is enhanced. Fiber gliding properties declined and adhesive properties increased when the oil concentration exceeded $0.6 \%$, causing the fibers to become sticky, resulting in being difficult to unwrap and card fiber as well as the emergence of winding roller and cylinder, and so on. These problems exerts negative impacts on fiber spinnability. 


\subsection{Effect of FFL-13 oil concentration on the carding process}

We use a carding machine to test PSA fibers. When the oil concentration was $0.6 \%$, the fiber carding process became easy, no winding of the pressing and steel rollers was observed, and a good web was obtained. Electrostatic testing showed that good spinnability is produced when the dynamic voltage of the combed cotton fiber is $800 \mathrm{~V}$. The PSA fibers could not constitute a continuous web when the oil concentration was not $0.6 \%$.

\subsection{STM analysis}

The PSA fiber dipped in different concentrations of FFL-13 oil was tested using STM and the results are shown in Figure 6. Figure $6(\mathrm{a}), 6(\mathrm{~b})$, and $6(\mathrm{c})$ shows gray-scale images of the fibers after dipping in oil at concentrations of $0.4 \%, 0.6 \%$, and $0.8 \%$, respectively. Moreover, Figure $6(\mathrm{~d}), 6(\mathrm{e})$, and $6(\mathrm{f})$ shows the fiber surfaces after processing by Photoshop and MATLAB. In these figures, points of the same height are joined together by straight lines or curves. The linearization technique showed other intersections, and red points indicate the highest points on the surface of the PSA fibers. Deep-blue points indicate the lowest points. Figure 6(a), 6(b), and 6(c) shows that the number of brightness stripes increases as the dipping concentration increases. In addition, the scans of the PSA fiber dipped in the oil concentration of $0.6 \%$ is satisfactory. Figure $6(d)$ and $6(f)$ shows a large number of red and blue points, and the oil spreads out unevenly over the fibers. Figure 6(e) shows oil spread out evenly over the fibers.

\subsection{The comparison of FFL-13 oil and $80 \mathrm{G} 33 \mathrm{NF}$}

The PSA fibers were dipped in the optimal oil concentration of $0.6 \%$. Table 1 shows that the specific resistance of FFL-13 oil and $80 \mathrm{G} 33 \mathrm{NF}$ are $4.6 \times 10^{7} \Omega \cdot \mathrm{cm}$ and $6.6 \times 10^{7} \Omega \cdot \mathrm{cm}$, respectively, because the longer alkyl chain length of phosphate, weaker hygroscopic property of phosphate, and $\mathrm{C}_{8}$ phosphate showed a stronger competitive adsorption of water in the air than $C_{12}$ phosphate [12]. After the use of the FFL-13 oil, F/F $\mu$ s and F/ F $\mu$ d of PSA fiber were 0.2113 and 0.1548 , respectively; and the $D$-value between $F / F \mu s$ and F/F $\mu d$ was 0.057 . After using 80G33NF, F/F $\mu$ s and F/F $\mu d$ of the PSA fiber were 0.2082 and 0.1574 , respectively, and the D-value between $F / F \mu s$ and $F /$ F $\mu d$ was 0.051 . FFL-13 oil can make PSA fiber with improved cohesion. The dynamic voltage and cohesion of FFL-13 oil and $80 \mathrm{G} 33 \mathrm{NF}$ are $800 \mathrm{~V}$ and $63.5 \mathrm{~N} / \mathrm{g}$ and $1000 \mathrm{~V}$ and $31.4 \mathrm{~N} / \mathrm{g}$, respectively. FFL-13 oil is superior to $80 \mathrm{G} 33 \mathrm{NF}$ in terms of specific resistance, dynamic voltage, and cohesion.

\subsection{FTIR spectra of FFL-13 oil and 80G33NF}

Figure 7 shows FTIR spectra of FFL-13 oil and 80 G33NF in the wavenumber range from $400 \mathrm{~cm}^{-1}$ to $4,000 \mathrm{~cm}^{-1}$. The curves clearly show that the $-\mathrm{CH}_{2}$ group stretching peak is at 2,923 $\mathrm{cm}^{-1},-\mathrm{OH}$ stretching peak at $3,353 \mathrm{~cm}^{-1},-\mathrm{NH}$ deformation peak at $1633 \mathrm{~cm}^{-1},-\mathrm{CH}$ deformation peak at $721 \mathrm{~cm}^{-1}$,
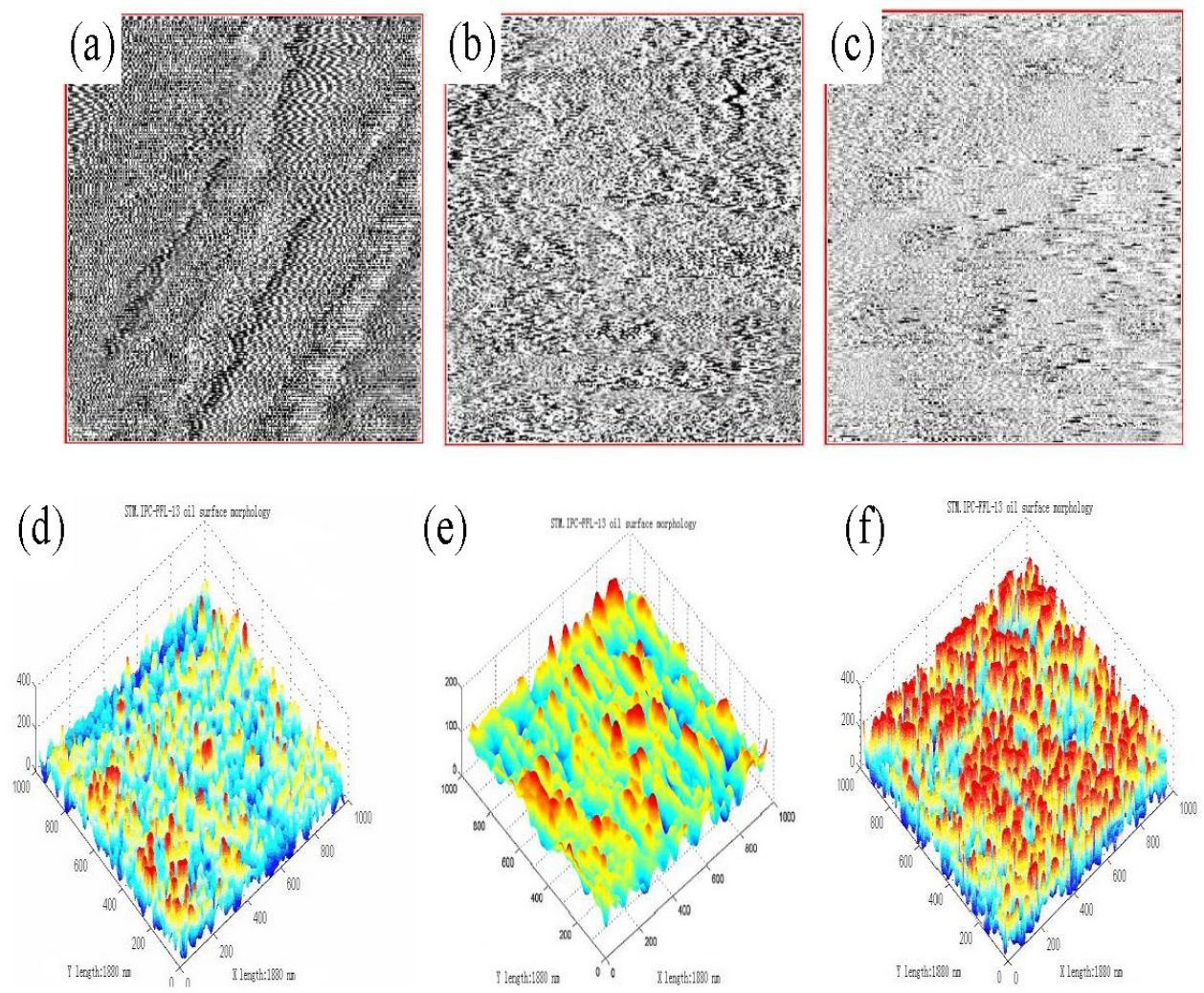

Figure 6. STM images and the surface morphology of PSA fiber: (a,d) oil concentration is $0.4 \%$, (b,e) oil concentration is $0.6 \%$, (c,f) oil concentration is $0.8 \%$. 
Table 1. Comparison of PSA fiber after treatment by FFL-13 oil and $80 \mathrm{G} 33 \mathrm{NF}$

\begin{tabular}{|c|c|c|c|c|c|c|}
\hline \multicolumn{3}{|c|}{ Sample } & $\begin{array}{l}\text { Number of } \\
\text { specimen }\end{array}$ & Mean & $\begin{array}{l}\text { Standard } \\
\text { deviation }\end{array}$ & P-value \\
\hline \multirow{2}{*}{$\begin{array}{c}\text { Specific } \\
\text { resistance } \\
(\Omega \cdot \mathrm{cm})\end{array}$} & \multicolumn{2}{|c|}{ FFL-13 } & 20 & $4.6 \times 10^{7}$ & $2.4 \times 10^{6}$ & 0.048 \\
\hline & \multicolumn{2}{|c|}{ 80G33NF } & 20 & $6.6 \times 10^{7}$ & $3.3 \times 10^{6}$ & 0.049 \\
\hline \multirow{4}{*}{$\begin{array}{l}\text { Friction } \\
\text { coefficient }\end{array}$} & \multirow{2}{*}{ FFL-13 } & $\mathrm{F} / \mathrm{F} \mu \mathrm{s}$ & 30 & 0.2113 & 0.0085 & 0.047 \\
\hline & & $\mathrm{F} / \mathrm{F} \mu \mathrm{d}$ & 30 & 0.1548 & 0.0077 & 0.049 \\
\hline & \multirow{2}{*}{ 80G33NF } & $\mathrm{F} / \mathrm{F} \mu \mathrm{s}$ & 30 & 0.2082 & 0.0105 & 0.048 \\
\hline & & $\mathrm{F} / \mathrm{F} \mu \mathrm{d}$ & 30 & 0.1574 & 0.0063 & 0.045 \\
\hline \multirow{2}{*}{$\begin{array}{l}\text { Dynamic } \\
\text { voltage } \\
\text { (V) }\end{array}$} & \multicolumn{2}{|c|}{ FFL-13 } & 30 & 800 & 32 & 0.049 \\
\hline & \multicolumn{2}{|c|}{$80 G 33 N F$} & 30 & 1,000 & 46 & 0.051 \\
\hline \multirow{2}{*}{$\begin{array}{c}\text { Cohesion } \\
(\mathrm{N} / \mathrm{g})\end{array}$} & \multicolumn{2}{|c|}{ FFL-13 } & 20 & 63.5 & 2.9 & 0.040 \\
\hline & \multicolumn{2}{|c|}{ 80G33NF } & 20 & 31.4 & 1.7 & 0.043 \\
\hline
\end{tabular}

carboxylate symmetric peak at $1,400 \mathrm{~cm}^{-1}$, and $\mathrm{C}-\mathrm{O}$ peak at $1,110 \mathrm{~cm}^{-1}$, except that the absorbent peak intensities are different. Compared with the 80G33NF, the significant decrease in the absorption peak at a wave number of $1,737 \mathrm{~cm}^{-1}$ and $2,854 \mathrm{~cm}^{-1}$ was attributed to the stretching vibration of $\mathrm{C}=\mathrm{O}$ and $-\mathrm{CH}_{2}$, which can be explained by the different chemical components of two oils. These changes induced the difference in performance of these two kinds of oils.

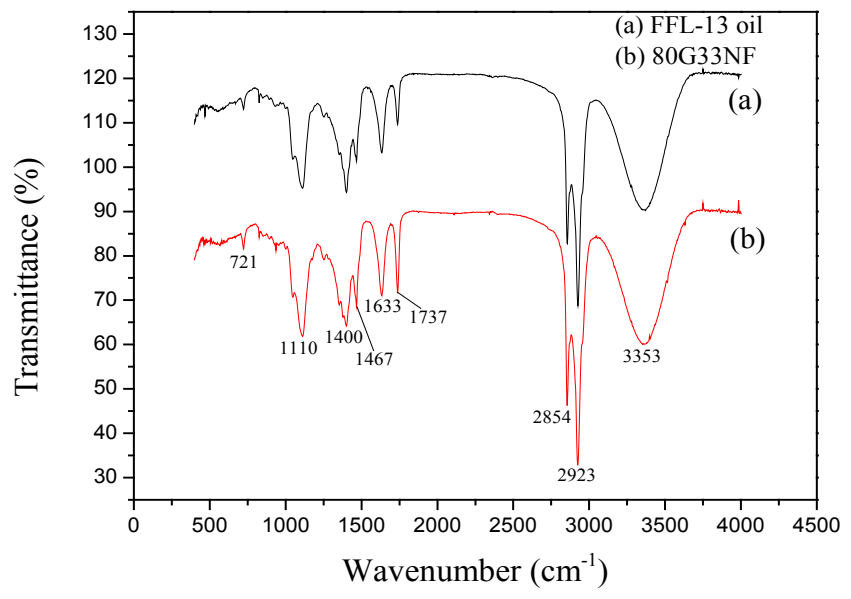

Figure 7. FTIR spectra of the FFL-13 oil and 80G33NF

\section{Conclusions}

In this study, the preparation of PSA fiber special oil is novel and has never been performed before. The experimental results can be summarized as follows:

(1) Fiber-specific resistance gradually decreased as the oil concentration, environment temperature, and humidity increased. If the oil concentration is too high, the oil film thickness on the fiber surface increases and the roller and sticky fibers intertwine, thereby causing difficulties during textile processing. Excess hygroscopic fibers also tend to adhere to the roller and other fibers, which affects spinnability.
(2) Fiber cohesion increased and fiber-gliding properties appeared to first increase and then decrease as the oil concentration increased. The oil spread out evenly on the fiber surface when the oil concentration was $0.6 \%$. At this point, the PSA fibers may be considered to achieve ideal gliding and cohesive properties.

(3) The specific resistance and friction properties of PSA fibers showed significant improvement and easy fiber carding when FFL-13 exclusive oil was used. These results provide that the oil markedly improves fiber spinnability.

\section{Acknowledgments}

This work was supported by Tianjin Science and Technology Plan Projects [No. 12TXGCCX01900].

\section{References}

[1] Wang, C. X., Lv, J. C., Gao, D. W., Liu, G. L., Jin, L. M., Liu, J. H. (2013). Surface modification and aging effect of polysulfonamide yarns treated by atmospheric pressure plasma. Fiber Polym, 14 (9), 1478-1484.

[2] Li, H. M., Zhu, Y., Xu, B., Wu, C. X., Zhao, J. X., Dai, M. $X$. (2013). Preparation and characterization of all paraposition polysulfonamide fiber. J Appl Polym Sci, 127, 342348.

[3] Zhou, J., Lin, L. T. (2010). Study on light stability property of polysulfonamide fiber. Tech Text, 2, 17-19.

[4] Ndlovu, L. N., Siddiqui, Q., Omollo, E., Yu, C. W. (2015). Physical properties of plain single jersey-knitted fabrics made from blended and core-spun polysulfonamide/cotton yarns. Physical Text. Res. J., 85(3), 262-271.

[5] Jia, X. L., Li, G., Yu, Y. H., Sui, G., Liu, H. Y., Li, Y. N., Li, P., Yang, X. P. (2009). Ablation and thermal properties of ethylene-propylene-diene elastomer composites reinforced with polysulfonamide short fibers. J Appl Polym Sci, 113, 283-289. 
[6] Jia, X. L., Yu, Y. H., Li, G., Sui, G., Li, P., Yang, X. P. (2010). Effects of curing systems and polysulfonamide pulp on the curing characteristics, mechanical properties, and swelling behavior of ethylene-propylene-diene elastomer composites. J Appl Polym Sci, 118, 1060-1067.

[7] Zou, C. L. (2010). Study on the spinning process of polysulfonamide yarn made by different fiber length, Master thesis, Donghua University, Shanghai.

[8] Li, X. H., Ao, L. M., Chen, Z. H. (2011). Production of polysulfonamide aramid 1313 blended yarn. Cotton Text Technol, 39 (3), 46-48.

[9] Dong, Z. W., Yang, J. P., Yu, C. W., Yin, Q. Y. (2010). Effect of polysulfonamide fiber length on yarn quality. Cotton Text Technol, 38 (3), 14-17.

[10] EL-SAYED, M. M., SANAD, S. H. (2007). The impact of new spinning technologies on the Egyptian cottons. Autex Res J, 8(4), 231-238.

[11] Huang, Y. P., Chen, T. K. (2007). Effect of surface treatment on pet spinning and the yarn property. Colloid Surface $A$, 295, 75-80.

[12] Zheng, G. (2006). Study on PET staple fiber finishes and fibers properties, Ph.D. thesis, Tianjin Polytechnic University, Tianjin.

[13] Tyagi, G. K. (2008). Influence of add-on spin finish on yarn quality in the $O E$ spinning of polyester fiber yarns. Indian $J$ Fibre Text Res, 33, 371-376.

[14] Dhamija, S., Tyagi, G. K., Kamar, D. (2000). Influence of spin finish and opening roller speed on the characteristics of OE friction-spun acrylic yarns. Indian J Fibre Text Res, 25, 169-176.
[15] Ammayappan, L., Sanjoy, D., Surajit, S. (2014). Effect of nano-polysiloxane based finishing on handle properties of jute blended fabric. Indian J Fibre Text Res, 39, 425-429.

[16] Fok, W. Y., Hild, D. N., Petrick, L. M., Obendorf, S. K. (2006). Autoxidation of Spin Finishes. Text Res J, 76(8), 614-618.

[17] Redston, J. P., Bernholz, W. F., Nahta, R. C. (1971). Emulsifier choice in design of spin finishes for man-made fibers. J Am Oil Chem Soc, 48(7), 344-348.

[18] Wan, A., Yu, W., Jiang, G. (2014). Pilling properties of wool single jersey made of compact and conventional ring yarns after anti-felting treatment. Text Res J, 84(7), 673-683.

[19] Zhou, C., Zheng, G., Liu, Y. J., Xu, J. Y. (2005). Development and application of new spinning finishes for large capacity PET staple fiber. J Text Res, 26(2), 85-88.

[20] Wang, C. X., Xu, H. L., Liu, Y., Qiu, Y. P. (2008). Influence of twist and filament location in a yarn on effectiveness of atmospheric pressure plasma jet treatment of filament yarns. Surf Coat Tech, 202, 2775-2782.

[21] Xu, H. Y. (2012). Development and application of spin finishes for polyester staple fiber, Master thesis, TianJin University, Tianjin.

[22] Zheng, G., Liu, Y. J., Xu, J. Y., Xiao, C. F. (2005). Polyester staple finishes and it's frictional property. J Text Res, 26, 85-87. 